\title{
Determinación de la eficiencia del aserrín y la fibra de coco utilizados como empaques para la remoción de contaminantes en Biofiltros para el tratamiento de aguas residuales
}

\section{(Determination of the efficiency of sawdust and coco fiber used as Biofilter for pollutant removal for the treatment of wastewater)}

\author{
Jimmy Vicente Reyes ${ }^{1}$
}

\begin{abstract}
Resumen:
El agua es un recurso muy utilizado por la humanidad tanto para uso industrial y doméstico, la cual se descarga en la red de alcantarillado público o pozos sépticos. El presente proyecto propone una alternativa ecológica de tratamiento de aguas residuales domésticas llamada Biofiltro, que se construye de material vivo (lombrices) e inerte (viruta y grava). Al irrigar el agua residual en este filtro biológico se ha demostrado alta eficiencia en la remoción de materia orgánica y organismos patógenos. El trabajo de campo se llevó a cabo con Biofiltros experimentales en los cuales, para obtener mejores condiciones se hizo variantes en la estructura del material inerte. Se realizaron dos Biofiltros pilotos, uno con empaque de aserrín y otro con empaque de fibra de coco en los cuales se hizo el tratamiento de agua residual doméstica, se tomaron muestras del efluente tratado en cada reactor y fueron sometidas a pruebas de laboratorio. El análisis e interpretación de resultados arrojaron que la eficiencia en la remoción de contaminantes del Biofiltro con aserrín es del 53.53 \% y está fuera de la norma, y del Biofiltro con fibra de coco es del $82.37 \%$ y está dentro de la norma de Calidad Ambiental y de Descarga de Efluentes: Recurso Agua.
\end{abstract}

Palabras clave: Tratamiento de aguas residuales, Biofiltro, Aserrín, Fibra de coco, Norma de calidad ambiental.

\begin{abstract}
:
Water is a resource used by mankind for industrial and domestic needs, which once used, is discharged into the public sewer system or septic tanks. This project proposes an ecological alternative for the treatment of wastewater from domestic use named Biofilter, which is built of living material (worms) and inert material (chip and gravel), which filters the wastewater; the biological filter has shown high efficiency in the removal of organic matter and pathogens. The field work was carried out with experimental biological filters, to ascertain the best composition of inert material, different variants were used. Two experimental Biofilters, one using sawdust and the other coco fiber were used in the treatment of domestic wastewater; treated samples from each reactor were subjected to laboratory analysis. The analysis and interpretation of results showed that the Biofilter using sawdust removed $53.53 \%$ of pollutants and is outside the required norm for wastewater treatment and the Biofilter using coco fiber removed $82.37 \%$ of contaminants and is within the Environmental Quality Norm and Effluent Discharge: Water Resource.
\end{abstract}

Keywords: Wastewater treatment, Biofilter, Sawdust, Coco fiber, Environmental Quality Norm.

\footnotetext{
${ }^{1}$ Universidad Tecnológica Equinoccial, Quito - Ecuador (vrja46470@ute.edu.ec)
} 


\section{Introducción}

El agua que se ha utilizado para alguna actividad u objetivo específico, cuando retorna al ambiente estará contaminada debido a la presencia de elementos extraños que se depositan en ellas.

Los contaminantes son diversos, pueden ser orgánicos e inorgánicos y debido a lo difícil de catalogar las ilimitadas combinaciones de productos químicos encontrados en aguas residuales, comúnmente se describen en pocas categorías generales (Castillo, 2005) (Masters \& Ela, 2008):

- Demanda de oxigeno: El material que demanda oxigeno es todo aquel que se oxida en el agua receptora y consume oxigeno molecular (comúnmente suele ser material orgánico biodegradable y ciertos compuestos inorgánicos).

- Nutrientes: Los nutrientes se consideran contaminantes cuando están presentes en cantidades excesivas debido a que perturban la red alimenticia. Las principales fuentes de nutrientes son detergentes, fertilizantes, excremento humano y animal.

- Microorganismos patógenos: En las aguas de desecho están presentes bacterias, virus y protozoos que excretaron personas o animales. Cuando estas son descargadas en cuerpos de agua se vuelven inapropiadas para beber e incluso insegura para nadar y pescar.

- Sólidos suspendidos: Son las partículas orgánicas e inorgánicas que arrastra el agua residual. Cuando reduce su velocidad, muchas de estas partículas se depositan en el fondo como sedimento y las que no se asientan provocan turbiedad.

- Sales minerales: Todo tipo de agua contiene cierta cantidad de sal, cuando la concentración de sales en el agua es elevada representa una amenaza para la biota animal y vegetal, el agua pierde utilidad para abastecer a la población o la irrigación.

- Compuestos tóxicos: Las aguas negras domésticas e industriales contienen sustancias tóxicas que si se descargan en grandes cantidades inutilizan cuerpos de agua. La persistencia de muchos compuestos tóxicos ha provocado que estos se concentren en la red alimenticia.

La FAO (2014), define el término "agua residual" a aquella "que no tiene valor inmediato, debido a su calidad' y resultan del uso de actividades domésticas o industriales, contiene contaminantes y gérmenes por lo cual se deben evacuar de manera segura para las personas y el ambiente. Las aguas residuales se clasifican en:

- Aguas residuales domésticas: son los residuos líquidos que se generan en viviendas y servicios que se transportan en el alcantarillado hacia una planta de tratamiento. 
- Aguas residuales industriales: son las aguas provenientes de las descargas de cualquier actividad comercial e industrial que no sean aguas residuales domésticas.

Con base al contenido de contaminantes se clasifican en:

- Aguas negras: son los residuos líquidos provenientes de inodoros (transportan excrementos y orina ricas en sólidos suspendidos, nitrógeno y coliformes fecales).

- Aguas grises: son los residuos líquidos provenientes de duchas, lavamanos y lavadoras que aportan sólidos suspendidos, fosfatos, grasas y coliformes.

A lo largo de la historia, las aguas residuales han sido consideradas como una molestia que debe eliminarse de manera barata y amigable con el ambiente. En años pasados, estos residuos se disponían en el sitio o se descargaban directamente en lagos o ríos, hechos que han ocasionado serios problemas de degradación ambiental, es por eso que, se han desarrollado diversos sistemas de tratamiento de aguas residuales.

Las aguas residuales se presentan sucias y contaminadas puesto que contienen materia orgánica e inorgánica debido a la presencia de ciertos compuestos y surge la necesidad de depurar o tratar estas aguas. Los tratamientos convencionales conducen las aguas residuales a una planta de tratamiento, sitio donde se remueven los contaminantes presentes mediante la utilización de diferentes métodos físicos, químicos y biológicos para devolver el agua a la naturaleza en las mejores condiciones posibles.

De acuerdo con Masters \& Ela (2008), "las plantas de tratamiento de aguas residuales se designan normalmente como de tratamiento primario, secundario, o avanzado, según el grado de purificación". En la Figura 1 se pueden observar las diferentes etapas que se llevan a cabo en plantas de tratamiento convencionales para la depuración de aguas residuales.

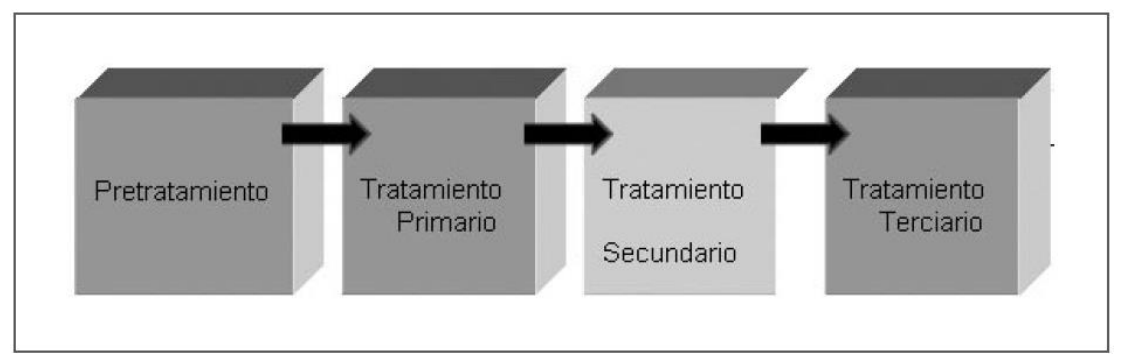

Figura 1. Etapas de tratamiento de aguas residuales

(Arana, 2009)

En la etapa de Pretratamiento las aguas residuales se conducen por una red de alcantarillado hasta la estación depuradora. Esta etapa consta de varias etapas:

- Desbaste: retención de sólidos gruesos.

- Desarenado: sedimentación de la arena por gravedad. 
- Desgrasado: suspensión de partículas de baja densidad, especialmente aceites y grasas.

La etapa de Tratamiento primario utiliza métodos físicos para eliminar la materia en suspensión y reducir la $\mathrm{DBO}$. Se distinguen varios procesos:

- Decantación: asentamiento de las partículas más densas por acción de la gravedad.

- Coagulación y floculación: rompe la suspensión y provoca aglomeración de partículas.

- Neutralización: corrige la excesiva acidez o alcalinidad del agua.

En la etapa de Tratamiento secundario aprovecha la capacidad de los microorganismos para que los residuos orgánicos se estabilicen en compuestos de baja energía. Explica Arana (2009), que el agua clarificada y depurada en la etapa de tratamiento biológico es transportada a la última etapa de tratamiento y que "contiene solo entre el 5 y 10 \% de la materia orgánica con la que entró".

En la última etapa, de Tratamiento avanzado, se busca eliminar los nutrientes y la materia orgánica no biodegradable mediante métodos físicos, químicos y/o biológicos para eliminar contaminantes específicos.

En nuestro país, especialmente en zonas rurales, se encuentran comunidades, pueblos e industrias que evacuan sus aguas servidas directamente a cuerpos de agua o las descargan en pozos sépticos sin que reciban tratamiento alguno puesto que no cuentan con estos sistemas, actividad que representa un riesgo potencial para la salud humana, los animales y el ambiente.

La presente investigación busca plantear como alternativa de remplazo a los pozos sépticos utilizados en zonas rurales a la tecnología no convencional de tratamiento de aguas residuales llamada Biofiltro que puede ser utilizada en hogares e industrias que no cuentan con servicio de alcantarillado público y que gracias a la actividad microbiológica degrada la materia orgánica y elimina contaminantes al esparcir el afluente contaminado en materiales orgánicos.

Un sistema no convencional de biotratamiento de aguas servidas y residuos industriales líquidos orgánicos (RILES) es el Lombrifiltro llamado también Biofiltro, que está constituido por distintos estratos filtrantes de materiales orgánicos e inorgánicos. Esta técnica fue desarrollada por el Dr. José Tohá Castellá y sus colaboradores en el Laboratorio de Biofísica de la Facultad de Ciencias Físicas y Matemática de la Universidad de Chile. El Biofiltro, es un tratamiento biológico que remueve; Coliformes Fecales, Sólidos Suspendidos, Sedimentables y Totales, DBO, Aceites y Grasas, Turbidez (Arango, 2003) (Guzmán, 2004).

El Sistema de Tratamiento de Aguas Servidas y RILES Biofiltro consta de las siguientes etapas:

- Pretratamiento físico: separación de sólidos, aceites y grasas.

- Tratamiento biológico: Biofiltro o Lombrifiltro. 
- Opcional: Desinfección por Radiación U.V. o Cloración. Depende de las características del efluente y solo en el caso de ser necesario.

El Biofiltro está compuesto de un medio filtrante y un soporte con varias capas de diferentes materiales. El medio filtrante es la capa superior compuesta de material orgánico, en este caso humus, en el cual habitan en gran cantidad lombrices rojas y microorganismos que digieren la materia orgánica retenida en esta capa, dejando al agua sin sus principales contaminantes. El soporte consta de dos capas, la primera de viruta que se encuentra a continuación de la capa de humus, $y$, la segunda que está formada de piedras de mediano tamaño asentadas sobre un falso fondo. Esta última capa provee soporte y aireación al sistema asegurando la permeabilidad del Biofiltro (Carmona, 2010).

Sugiere Hernández (2005), que " $1 \mathrm{~m}^{2}$ efectivo de Biofiltro para tratar $1 \mathrm{~m}^{3}$ de aguas servidas diarias" y añade que "para tratar $1 \mathrm{~m}^{3}$ de residuos industriales líquidos se requerirá mayor superficie, debido a los parámetros contaminantes que posee".

\section{Materiales y métodos}

La presente investigación está orientada en beneficio de los trabajadores de la Central Termoeléctrica Sacha de Termopichincha EP, planta que está ubicada en el cantón Sacha en la provincia de Orellana y genera 20.4 MW de energía eléctrica. Tiene 39 trabajadores que disponen de $3 \mathrm{~m}^{3}$ diarios de agua, la cual es captada de un pozo y es destinada para actividades de cocina, duchas y servicios higiénicos; se consumen 45 litros por trabajador, y todo el personal genera 1.8 $\mathrm{m}^{3}$ diarios de agua residual que se descargan en un pozo séptico construido de hormigón armado con capacidad de almacenamiento de $10 \mathrm{~m}^{3}$ y que en los últimos meses ha tenido varios problemas de funcionamiento debido a desalojos continuos por saturación rápida, desbordamientos cuando filtran las aguas de procedencia pluvial y malos olores.

El Biofiltro experimental consta de un Tanque homogenizador experimental cuyo objetivo es almacenar y homogenizar la muestra del agua residual. El tanque de homogenización, como se detalla en la Figura 2, tiene capacidad de 25 litros, de forma cilíndrica y material de plástico con una red de cañería para el desalojo del residuo hacia la próxima etapa de la planta piloto de dimensiones de $16 \mathrm{~cm}$ de radio y $32 \mathrm{~cm}$ de largo. 


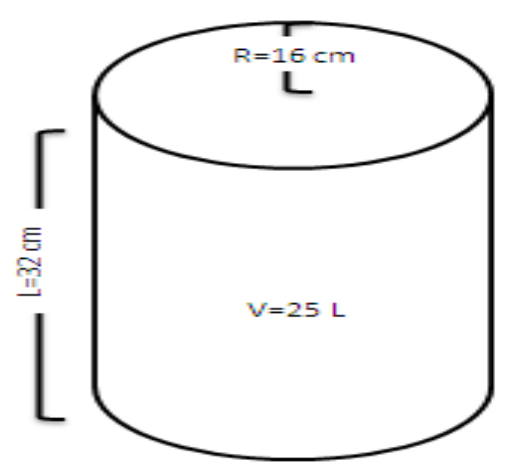

Figura 2. Tanque homogenizador experimental

El sedimentador experimental tiene el objetivo de retener sólidos de la muestra del agua residual. El sedimentador, como se detalla en la Figura 3, de forma cuadrada y material acrílico con la capacidad de almacenar 3 litros con una red de cañería para la recepción y desalojo del residuo hacia la próxima etapa de la planta piloto de dimensiones de $15 \mathrm{~cm}$ de largo, $15 \mathrm{~cm}$ de ancho y 15 $\mathrm{cm}$ de alto.

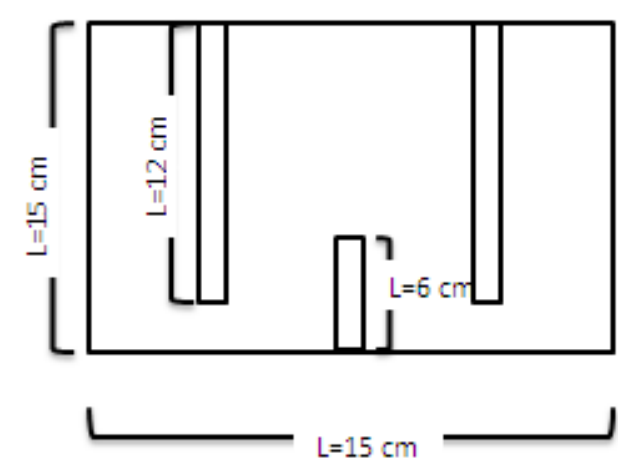

Figura 3. Sedimentador experimental

El Biofiltro experimental cuyo objetivo es depurar la muestra de agua residual. El Biofiltro, como se podrá observar en la Figura 4, de forma rectangular y material acrílico con capacidad de tratar 6 litros con una red de cañería de entrada del afluente y desalojo del efluente de la planta piloto de dimensiones de $30 \mathrm{~cm}$ de largo, $10 \mathrm{~cm}$ de ancho y $30 \mathrm{~cm}$ de alto.

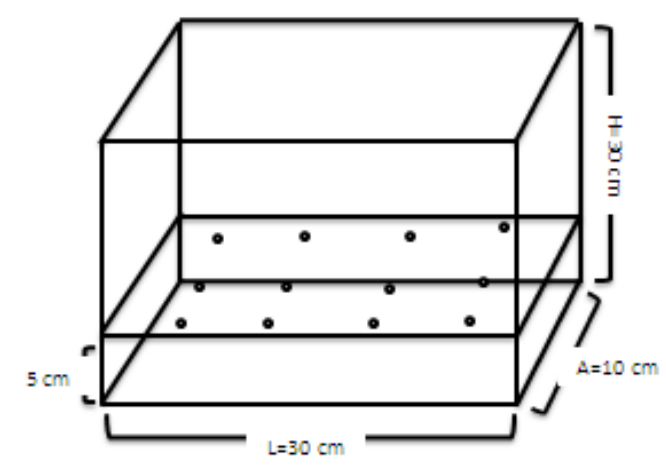

Figura 4. Sedimentador experimental

El sistema piloto de tratamiento de aguas residuales para la experimentación, utilizó los materiales y equipos detallados en la Tabla 1 que fueron necesarios para depurar el agua residual a fin de obtener resultados del proceso de tratamiento. 
La etapa de experimentación se realizó aledaña al pozo séptico que recepta el agua residual para la recolección de la muestra, el tanque de almacenamiento experimental se colocó sobre una base a $30 \mathrm{~cm}$ de altura a fin que el flujo del agua a tratar se conduzca por gravedad hacia la siguiente etapa de tratamiento, también se instaló una red de cañería para el desalojo del residuo líquido en el tanque de almacenamiento. La cañería de desalojo se conectó con el sedimentador y el Biofiltro para que el líquido fluya por todos los componentes del sistema depurador piloto.

Se colocaron los estratos filtrantes y las lombrices rojas en el soporte del Biofiltro, se revisaron los equipos con una prueba en blanco con agua limpia con el fin de determinar el buen funcionamiento, se verificó que los equipos y materiales funcionen de manera correcta para proceder a iniciar el tratamiento del agua residual en la planta piloto.

Tabla 1. Materiales y equipos utilizados en el proceso de experimentación

\begin{tabular}{ccccc}
\hline Cantidad & Unidad & Descripción & Material & Función \\
\hline 1 & $\mathrm{~kg}$ & Estrato filtrante & Grava & Retención de materia orgánica \\
1 & $\mathrm{~kg}$ & Estrato filtrante & Humus & Alberga a las lombrices rojas \\
1 & $\mathrm{U}$ & Tanque de & Acrílico & Homogenización de residuo liquido \\
1 & $\mathrm{U}$ & Sedimentamador & Acrílico & Retención sólidos \\
1 & $\mathrm{U}$ & Biofiltro & Acrílico & Soporte de estratos filtrantes \\
1 & $\mathrm{U}$ & Termómetro & Vidrio & Medición de temperatura del agua residual \\
1 & $U$ & Jarra & Plástico & Recolección de agua liquido \\
1 & $\mathrm{U}$ & Malla & Yute & Soporte de lombrices \\
1 & $\mathrm{U}$ & Embudo & Plástico & Recolección de agua residual \\
2 & $\mathrm{~kg}$ & Estrato filtrante & Aserrín & Retención de materia orgánica \\
2 & $\mathrm{~kg}$ & Estrato filtrante & Fibra de coco & Retención de materia orgánica \\
5 & $\mathrm{U}$ & Peachimetro & Papel & Medición de pH del agua residual \\
22 & $\mathrm{U}$ & Frascos & Vidrio & Recolección de aguas residuales \\
40 & $\mathrm{U}$ & Lombriz roja & N/A & Organismo depurador \\
50 & $\mathrm{~L}$ & Agua residual & Líquido & Sustrato para las lombrices \\
\hline
\end{tabular}

Las variables de diseño de la planta piloto fueron:

- Área del reactor: $0.03 \mathrm{~m}^{2}$

- Lombrices rojas: 40 unidades

- Volumen reactor: 6 litros

- Tiempo de residencia: 90 minutos

- Caudal del residuo: $70 \mathrm{ml} / \mathrm{min}$

El tratamiento en la planta piloto con estrato filtrante de aserrín se realizó en dos períodos, de 10:00 a 11:30 y de 14:00 a 15:30. El tratamiento en la planta piloto con estrato filtrante de fibra de coco se realizó también en dos períodos, de 10:30 a 12:00 y de 14:30 a 16:00.

En la Tabla 2 se describen los componentes utilizados en los Biofiltros experimentales 1 y 2 :

Tabla 2. Componentes utilizados en el Biofiltro Experimental 1

\begin{tabular}{cccc}
\hline Material & Cantidad & Unidad & Función \\
\hline Lombrices rojas & 40 & $\mathrm{U}$ & Organismo depurador \\
Grava & 1500 & $\mathrm{~cm}^{3}$ & Retención de materia orgánica \\
Humus & 300 & $\mathrm{~cm}^{3}$ & Alberga a las lombrices \\
Aserrín y viruta * & 4200 & $\mathrm{~cm}^{3}$ & Retención de materia orgánica \\
Fibra de coco ** & 4200 & $\mathrm{~cm}^{3}$ & Retención de materia orgánica \\
\hline
\end{tabular}

Empaque de aserrín y viruta utilizado únicamente en el Biofiltro experimental 1.

** Empaque de coco utilizado únicamente en el Biofiltro experimental 2. 
Para el Biofiltro experimental 1 se utilizó como empaque; aserrín y viruta común de aserradero puesto que esta materia es la más empleada en estos sistemas de tratamiento debido a su fácil adquisición y bajos costos mientras que para el Biofiltro experimental 2 se realizó una variante a este sistema de tratamiento, se utilizó fibra de coco, que constituye una opción de material para ser usado como empaque.

El proceso de experimentación se inició tomando la muestra de agua residual y colocándola en el Tanque Homogenizador, mezclada la muestra y por gravedad, el agua a ser tratada fue conducida hacia el Tanque Sedimentador para retener sólidos y otros materiales que puedan afectar el buen funcionamiento del Biofiltro. Completada la etapa anterior, el flujo de agua residual pasó hacia el Biofiltro para la retención y degradación de la materia orgánica por parte de las lombrices rojas que se encontraban en el empaque superior del biorreactor mientras que el agua continuaba atravesando los empaques de aserrín/viruta del Biofiltro Experimental 1 como se observa en la Figura 5 (a) y el de fibra de coco correspondiente al Biofiltro Experimental 2 como se observa en la Figura 5 (b). Finalmente, el efluente tratado fue desalojado por la parte inferior del reactor hacia un colector aledaño a la planta piloto.

a)

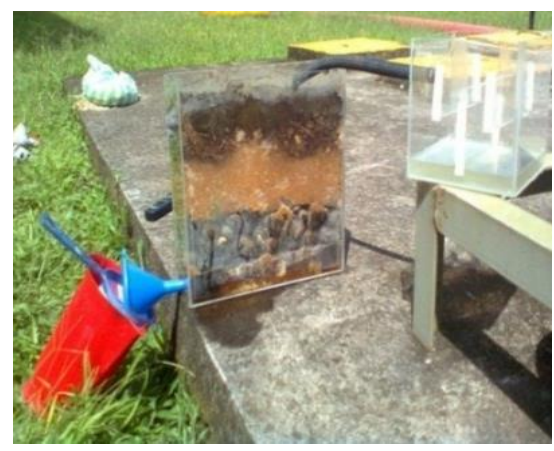

b)

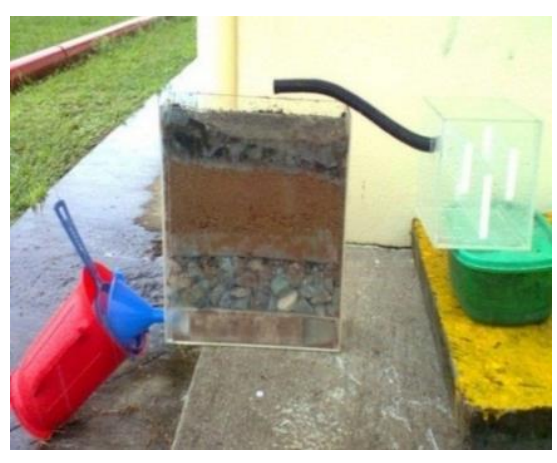

Figura 5. Tratamiento del agua residual en el Biofiltro experimental 1 y 2

Los períodos de tratamiento se desarrollaron con normalidad durante 90 minutos cada uno, al finalizar este tiempo se realizaron los muestreos que abarcaron su recolección de manera técnica a la salida del efluente en la planta piloto. A las 11:30 y 15:30 horas se procedió a recolectar las muestras del agua tratada en el Biofiltro experimental 1, mientras que a las 12:00 y 16:00 horas se recolectaron las muestras del agua tratada en el Biofiltro experimental 2. Los parámetros de Temperatura y $\mathrm{pH}$ se midieron In situ, consecutivamente a esta medición, la muestra recolectada fue rotulada y preservada en un cooler hasta llegar al laboratorio para su análisis.

\section{Resultados}

En Ecuador, el Texto Unificado de Legislación Secundaria del Ministerio del Ambiente (TULSMA) (2003) es el instrumento legal aplicable a la siguiente investigación, que corresponde a la Tabla 12 del Anexo 1 A Norma de Calidad Ambiental y Descarga de Efluentes: Recurso Agua, Libro VI De 
La Calidad Ambiental. En la Tabla 3 se detallan los límites máximos permisibles establecidos para las descargas líquidas a un cuerpo de agua dulce.

El análisis e interpretación de resultados de los reportes de laboratorio de las muestras recolectadas en los experimentos realizados, se detalla en la Tabla 4 por tipo de estrato filtrante, tanto del afluente como de los efluentes y su cumplimiento o no comparado con los límites máximos permisibles de la Norma de Calidad Ambiental y Descarga de Efluentes: Recurso Agua del TULSMA.

Tabla 3. Límites de descarga a un cuerpo de agua dulce

\begin{tabular}{cccc}
\hline PARÁMETROS & $\begin{array}{c}\text { EXPRESADO } \\
\text { COMO }\end{array}$ & UNIDAD & $\begin{array}{c}\text { LÍMITE MÁXIMO } \\
\text { PERMISIBLE }\end{array}$ \\
\hline Potencial de hidrógeno & $\mathrm{pH}$ & - & $5-9$ \\
Temperatura & ${ }^{\circ} \mathrm{C}$ & - & $<35$ \\
$\begin{array}{c}\text { Sólidos Suspendidos } \\
\text { Totales }\end{array}$ & - & $\mathrm{mg} / \mathrm{l}$ & 100 \\
$\begin{array}{c}\text { Sólidos Totales } \\
\text { Demanda Química de } \\
\text { Oxígeno }\end{array}$ & - & $\mathrm{mg} / \mathrm{l}$ & 1600 \\
$\begin{array}{c}\text { Demanda Bioquímica de } \\
\text { Oxígeno (5 días) } \\
\text { Hierro Total }\end{array}$ & D.B.O. & $\mathrm{mg} / \mathrm{l}$ & 250 \\
Coliformes Fecales & Fe & $\mathrm{mg} / \mathrm{l}$ & 100 \\
Aceites y grasas & $\begin{array}{c}\text { Sustancias } \\
\text { solubles en } \\
\text { hexano }\end{array}$ & $\mathrm{mg} / \mathrm{l}$ & 10 \\
\hline
\end{tabular}

Tabla 4. Detalle de caracterización del afluente y efluentes de los Biofiltros experimentales 1 y 2

\begin{tabular}{ccccccc}
\hline Parámetro & Unidad & Afluente & $\begin{array}{c}\text { Efluente } \\
\text { Biofiltro 1 }\end{array}$ & $\begin{array}{c}\text { Eficiencia } \\
\text { Biofiltro 1 }\end{array}$ & $\begin{array}{c}\text { Efluente } \\
\text { Biofiltro 2 }\end{array}$ & $\begin{array}{c}\text { Eficiencia } \\
\text { Biofiltro 2 }\end{array}$ \\
\hline Temperatura & ${ }^{\circ} \mathrm{C}$ & 27 & 27 & Cumple & 26 & Cumple \\
\hline $\mathrm{Ph}$ & --- & 6.2 & 5.8 & Cumple & 6.9 & Cumple \\
\hline Sólidos suspendidos totales & $\mathrm{mg} / /$ & 223 & 370 & No Cumple & 40 & $82.06 \%$ \\
\hline Sólidos totales & $\mathrm{mg} / /$ & 757 & 3264 & No Cumple & 603 & $20.34 \%$ \\
\hline Coliformes fecales & $\mathrm{NMP} / 100 \mathrm{ml}$ & $1,00 \mathrm{E}+07$ & $1,10+\mathrm{E} 05$ & $98.90 \%$ & $1,10+\mathrm{E} 05$ & $98.90 \%$ \\
\hline Aceites y Grasas & $\mathrm{mg} / \mathrm{l}$ & 50 & 0.3 & $99.40 \%$ & 0.3 & $99.40 \%$ \\
\hline DQO & $\mathrm{mg} / \mathrm{l}$ & 583 & 3650 & No Cumple & 174 & $70.15 \%$ \\
\hline DBO & $\mathrm{mg} / /$ & 200 & 39 & $80.50 \%$ & 23 & $88.50 \%$ \\
\hline Hierro & $\mathrm{mg} / /$ & 160 & 6.6 & $95.88 \%$ & 0.19 & $99.88 \%$ \\
\hline
\end{tabular}

El análisis del parámetro Temperatura de acuerdo con los resultados de laboratorio como se

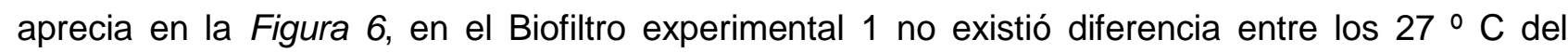


afluente y los $27^{\circ} \mathrm{C}$ del efluente, mientras que en el Biofiltro experimental 2 existió un descenso


parámetro se encuentra dentro de la norma ambiental aplicable que permite en descargas líquidas hasta $35 \stackrel{\circ}{C}$.

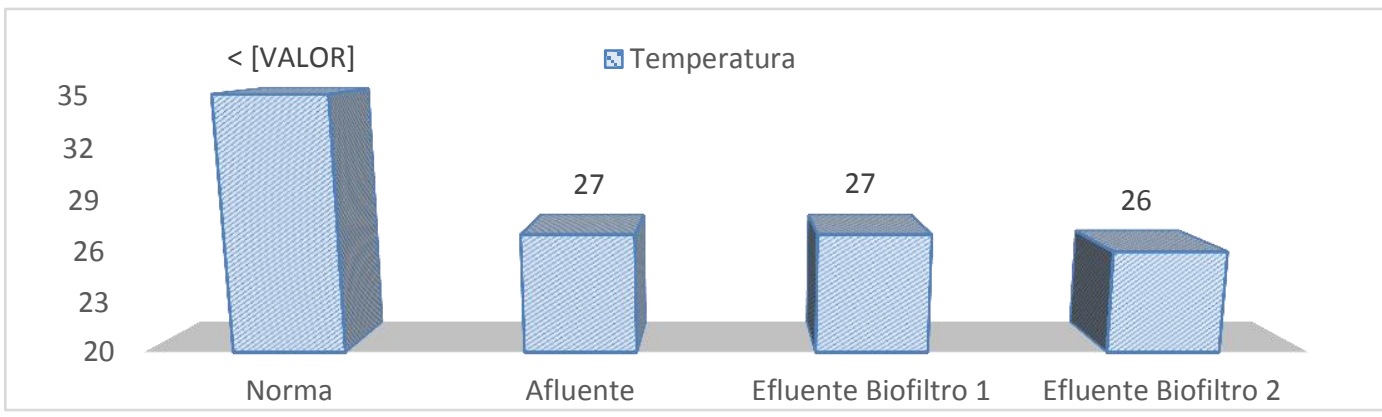

Figura 6. Análisis de parámetro Temperatura en los Biofiltros experimentales 1 y 2

El análisis del parámetro Potencial Hidrógeno de acuerdo con los resultados de laboratorio como se aprecia en la Figura 7, existió una diferencia mínima entre los 6.2 del afluente y los 5.8 del efluente en el Biofiltro experimental 1 mientras que hubo un ligero aumento de la variable con 6.9 en el efluente respecto a los 6.2 del afluente en el Biofiltro experimental 2. En ambos Biofiltros este parámetro se encuentra dentro de la norma ambiental aplicable que permite valores de 5 a 9 en descargas líquidas.

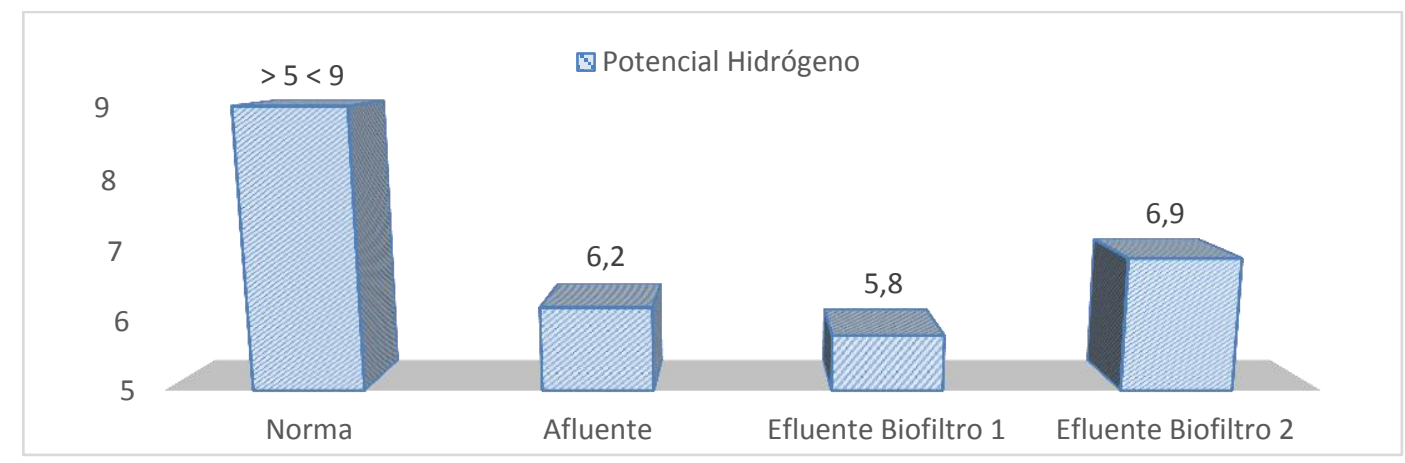

Figura 7. Análisis de parámetro Potencial Hidrógeno en los Biofiltros experimentales 1 y 2

El análisis del parámetro Sólidos Suspendidos Totales de acuerdo con los resultados de laboratorio se puede apreciar en la Figura 8, existió incremento en $370 \mathrm{mg} / \mathrm{l}$ del efluente con respecto a los $223 \mathrm{mg} / \mathrm{l}$ del afluente debido a la presencia de restos del empaque que se han disgregado por lo que este parámetro se encuentra fuera de la norma ambiental en el Biofiltro experimental 1. En el Biofiltro experimental 2 entre los $223 \mathrm{mg} / \mathrm{l}$ del afluente existió $40 \mathrm{mg} / \mathrm{l}$ de remoción en el efluente, siendo $82.06 \%$ el porcentaje de retención de sólidos en el tratamiento de la degradación de materia orgánica por lo cual se encuentra dentro de la norma ambiental aplicable que permite hasta $100 \mathrm{mg} / \mathrm{l}$ en descargas líquidas. 


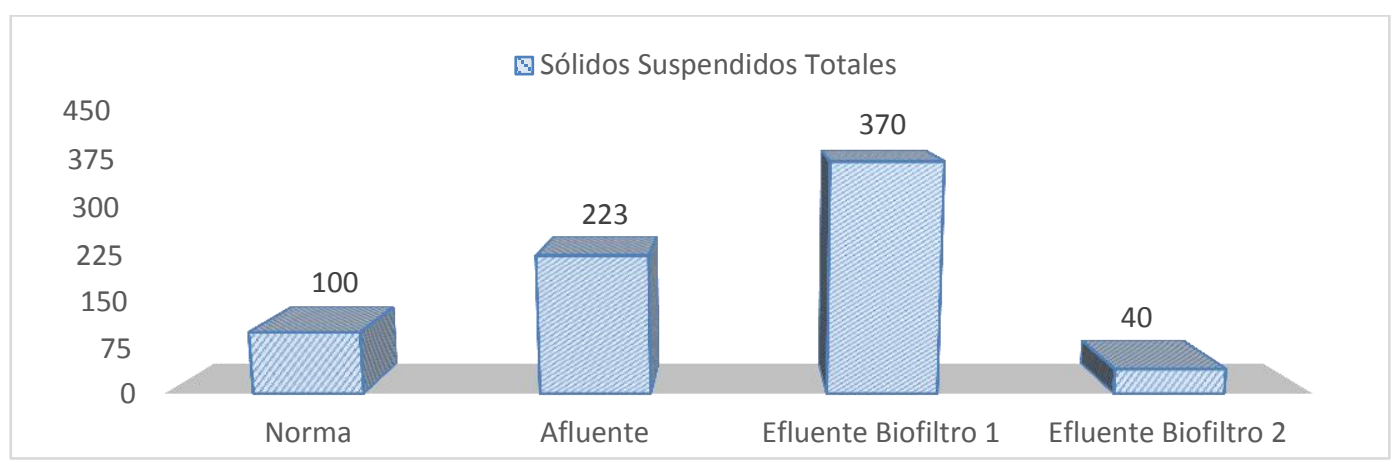

Figura 8. Análisis de parámetro Sólidos Suspendidos Totales en Biofiltros experimentales 1 y 2

El análisis del parámetro Sólidos Totales de acuerdo con los resultados de laboratorio se puede apreciar en la Figura 9, en el Biofiltro experimental 1 existió aumento de $3264 \mathrm{mg} / \mathrm{l}$ en el efluente con respecto a los $757 \mathrm{mg} / \mathrm{l}$ del afluente debido a la presencia de restos del empaque que no se han compactado de manera correcta por lo cual se han disgregado y se encuentra fuera de la norma. En el Biofiltro experimental 2, entre los $757 \mathrm{mg} / \mathrm{l}$ del afluente existió $603 \mathrm{mg} / \mathrm{l}$ de remoción en el efluente, donde $20.34 \%$ es el porcentaje de remoción en el tratamiento de la degradación de materia orgánica por lo cual se encuentra dentro de la norma ambiental aplicable que permite hasta $1600 \mathrm{mg} / \mathrm{l}$ en descargas líquidas.

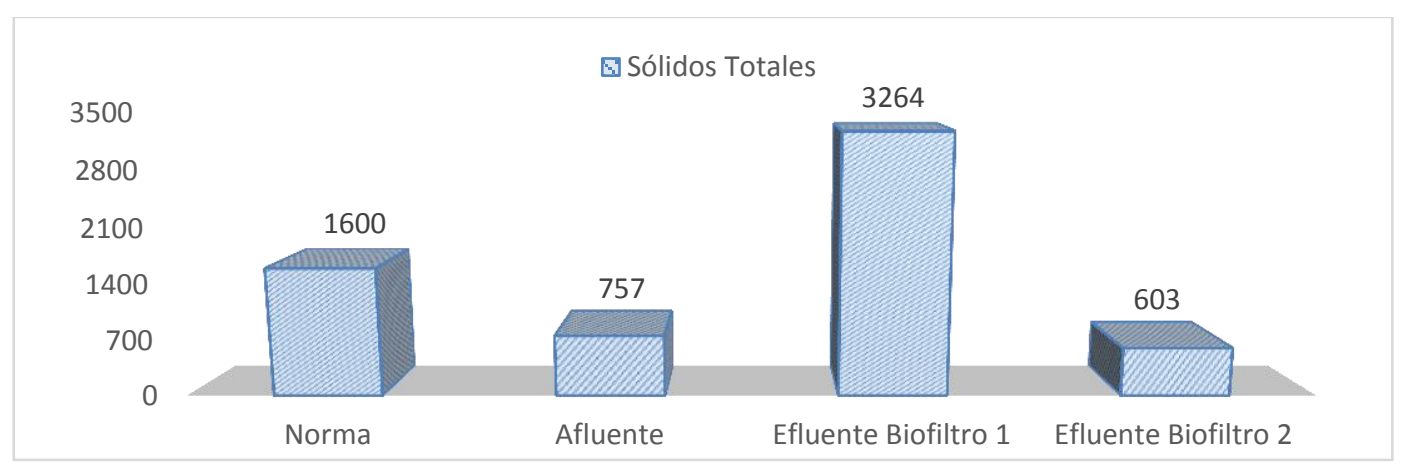

Figura 9. Análisis de parámetro Sólidos Totales en los Biofiltros experimentales 1 y 2

El análisis del parámetro Coliformes Fecales de acuerdo con los resultados de laboratorio se puede apreciar en la Figura 10 que tanto en el Biofiltro experimental 1 como en el 2, entre los 1,00E+07 NMP/100 ml del afluente y los 1,10+E05 NMP/100 ml del efluente, existió $98.90 \%$ de remoción de Coliformes Fecales y a pesar del elevado porcentaje de remoción que se encuentran fuera de la norma ambiental aplicable puesto que esta exige una remoción mayor al $99.99 \%$. 




Figura 10. Análisis de parámetro Coliformes Fecales en los Biofiltros experimentales 1 y 2

El análisis del parámetro Aceites y Grasas de acuerdo con los resultados de laboratorio se puede apreciar en la Figura 11 que tanto en el Biofiltro experimental 1 como en el 2, entre los $50 \mathrm{mg} / \mathrm{l}$ del afluente y los $<0.3 \mathrm{mg} / \mathrm{l}$ del efluente, existió $99.40 \%$ de remoción de este parámetro por lo cual se encuentra dentro de la norma ambiental aplicable puesto que se encuentran bajo los $0.3 \mathrm{mg} / \mathrm{l}$ para descargas líquidas.

El análisis del parámetro Demanda Química de Oxígeno de acuerdo con los resultados de laboratorio se puede apreciar en la Figura 9, existió un aumento de $3650 \mathrm{mg} / \mathrm{l}$ en el efluente con respecto a los $583 \mathrm{mg} / \mathrm{l}$ del afluente debido a la descomposición de la materia orgánica donde existe liberación de energía y se encuentra fuera de norma en el Biofiltro experimental 1. En el Biofiltro experimental 2 entre los $583 \mathrm{mg} / \mathrm{l}$ del afluente existieron $174 \mathrm{mg} / \mathrm{l}$ de remoción en el efluente, siendo $70.15 \%$ porcentaje de remoción y se encuentra dentro de la norma ambiental aplicable que permite hasta $250 \mathrm{mg} / \mathrm{l}$ en descargas líquidas.

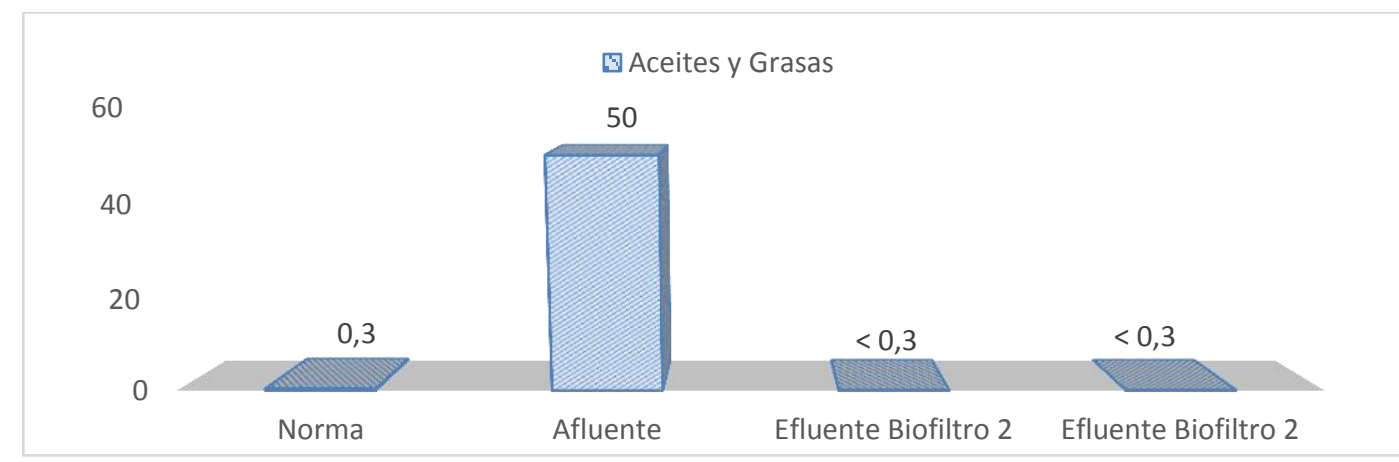

Figura 11. Análisis de parámetro Aceites y Grasas en los Biofiltros experimentales 1 y 2

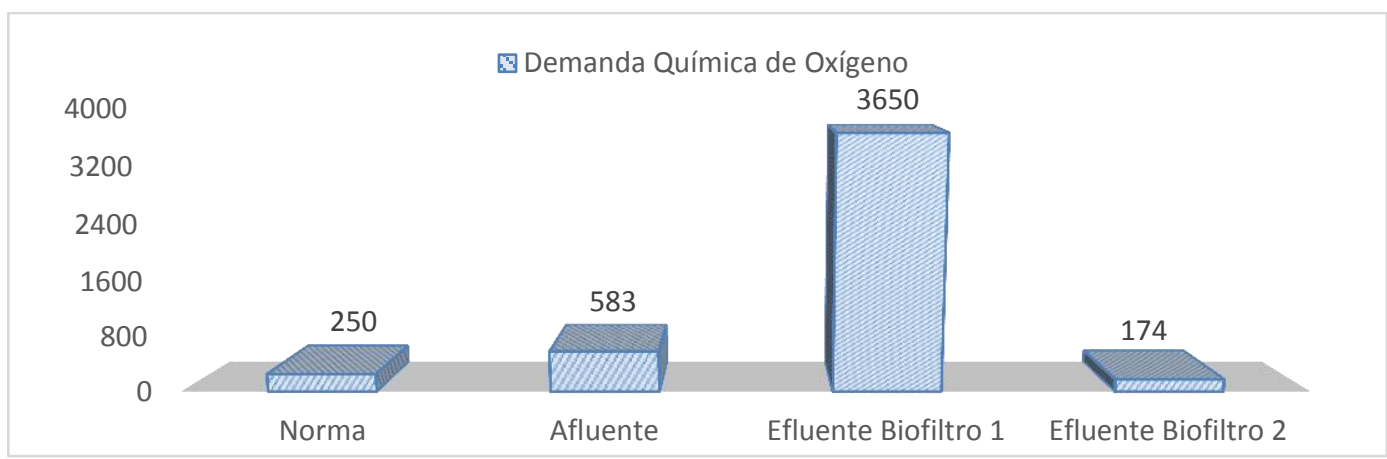

Figura 12. Análisis de parámetro Demanda Química de Oxígeno en Biofiltros experimentales 1 y 2 
El análisis del parámetro Demanda Bioquímica de Oxígeno de acuerdo con los resultados de laboratorio, en el Biofiltro experimental 1 entre los $200 \mathrm{mg} / \mathrm{l}$ del afluente y los $39 \mathrm{mg} / \mathrm{l}$ del efluente, existió $80.50 \%$ de remoción de este parámetro mientras que en el Biofiltro experimental 2, entre los $200 \mathrm{mg} / \mathrm{l}$ del afluente y los $23 \mathrm{mg} / \mathrm{l}$ del efluente, existió $88.50 \%$ de remoción de este parámetro en el tratamiento de la degradación de materia orgánica. Como se aprecia en la Figura 13, en ambos Biofiltros este parámetro se encuentra dentro de la norma ambiental aplicable que permite en descargas líquidas hasta $100 \mathrm{mg} / \mathrm{l}$.

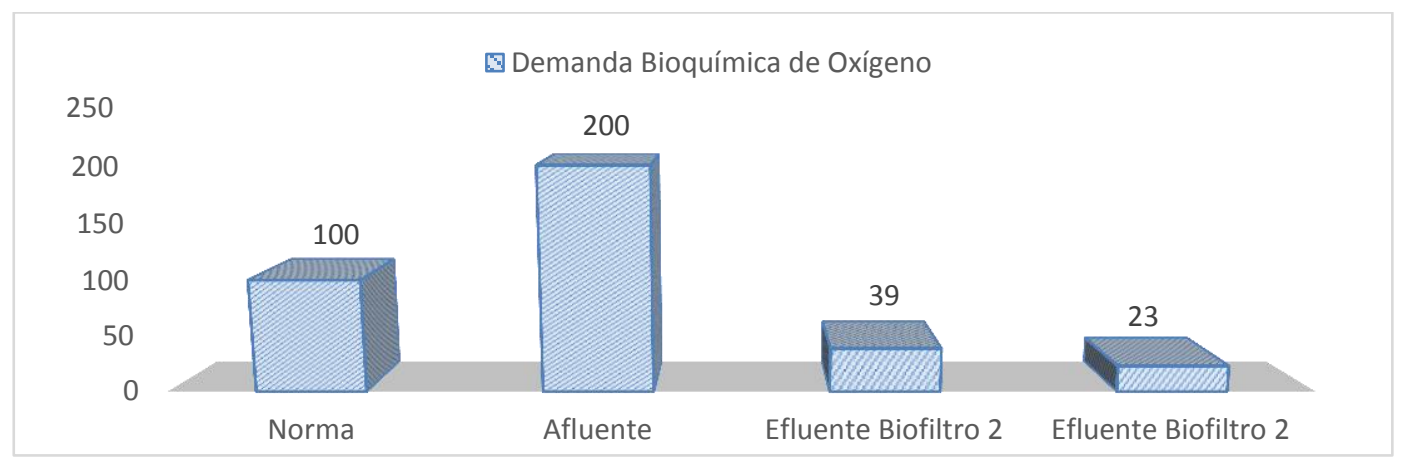

Figura 13. Análisis de parámetro Demanda Bioquímica de Oxígeno en los Biofiltros experimentales 1 y 2

El análisis del parámetro Hierro de acuerdo con los resultados de laboratorio, en el Biofiltro experimental 1 entre los $160 \mathrm{mg} / \mathrm{l}$ del afluente y los $6.6 \mathrm{mg} / \mathrm{l}$ del efluente, existió $95.88 \%$ de remoción de este parámetro mientras que en el Biofiltro experimental 2, entre los $160 \mathrm{mg} / \mathrm{l}$ del afluente y los $0.19 \mathrm{mg} / \mathrm{l}$ del efluente, existió 99.88 \% de remoción de este parámetro en el tratamiento de la degradación de materia orgánica. Como se aprecia en la Figura 14, en ambos Biofiltros este parámetro se encuentra dentro de la norma ambiental aplicable que permite en descargas líquidas hasta $10 \mathrm{mg} / \mathrm{l}$.

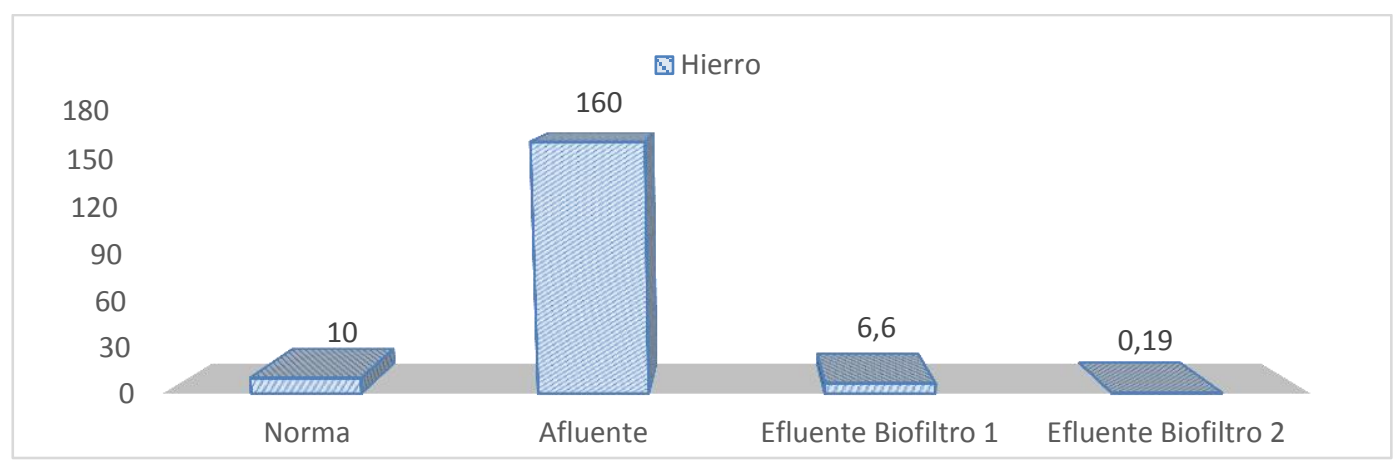

Figura 14. Análisis de parámetro Hierro en los Biofiltros experimentales 1 y 2

\section{Discusión}

Los resultados de todos los parámetros monitoreados en el proceso de tratamiento experimental, el análisis correspondiente al afluente del agua residual hacia la planta piloto de tratamiento, en el que se evidencia que los parámetros más importantes; Temperatura, $\mathrm{pH}$, Sólidos Suspendidos, 
Sólidos Totales, Cloro, Aceites y Grasas, DBO, DQO y Hierro, con resultados fuera de la norma aplicable.

Los resultados de todos los parámetros monitoreados en el proceso de tratamiento experimental, el análisis que corresponde al efluente del agua tratada en la planta piloto de tratamiento con empaque de aserrín/viruta, en el cual se evidencia que los parámetros más importantes; Temperatura, pH, Cloro, Aceites y Grasas, DBO y Hierro, con resultados dentro de la norma y los Sólidos Suspendidos, Sólidos Totales y DQO, con resultados fuera de la norma aplicable.

Referente a los parámetros de Sólidos Suspendidos y Sólidos Totales, se advierte un aumento en el caudal del efluente con respecto al afluente, debido a la presencia de restos del empaque que se han disgregado del Biofiltro experimental 1. Referente al parámetro DQO, se evidencia un aumento en el caudal del efluente con respecto al afluente, debido a la descomposición de la materia orgánica donde existe liberación de energía en el Biofiltro.

En el Biofiltro experimental 1 se utilizó como empaque; aserrín y viruta de madera, y, los análisis de resultados arrojaron que la eficiencia de este Biofiltro experimental fue de $53.53 \%$ en la remoción de contaminantes.

Los resultados de todos los parámetros monitoreados en el proceso de tratamiento experimental, el análisis correspondiente al efluente del agua tratada en la planta piloto de tratamiento con empaque de fibra de coco, en el cual se evidencia que los parámetros más importantes; Temperatura, pH, Sólidos Suspendidos, Sólidos Totales, Cloro, Aceites y Grasas, DBO, DQO y Hierro, con resultados dentro de la norma.

En el Biofiltro experimental 2 se utilizó como variante de empaque; fibra de coco, y, los resultados arrojaron que la eficiencia de este Biofiltro experimental fue de $82.37 \%$ en la remoción de contaminantes.

\section{Conclusiones y recomendaciones}

En las zonas rurales del país, la solución más popular para el tratamiento de aguas residuales en comunidades e industrias que no cuentan con el servicio público de alcantarillado, es realizar descargas de sus aguas residuales en pozos sépticos. El Biofiltro es una técnica que tiene poca o nula aplicación en nuestro país debido a los pocos estudios que se han realizado de este sistema en el tratamiento de aguas residuales.

Las características físicas, químicas y biológicas del agua residual doméstica de acuerdo con los informes de laboratorio, alcanzó parámetros fuera de la norma ambiental: $\mathrm{pH}=6.2$, Sólidos Suspendidos Totales $=223 \mathrm{mg} / \mathrm{l}$, Sólidos Totales $=757 \mathrm{mg} / \mathrm{l}$, Cloro $=60 \mathrm{mg} / \mathrm{l}$, Colonias $=1 \times 10^{7}$ $\mathrm{NMP} / 100 \mathrm{ml}, \mathrm{A} \& \mathrm{G}=50 \mathrm{mg} / \mathrm{l}, \mathrm{DQO}=583 \mathrm{mg} / \mathrm{l}, \mathrm{DBO}=200 \mathrm{mg} / \mathrm{l}$, Hierro = $160 \mathrm{mg} / \mathrm{l}$, lo que justifica el tratamiento de estas aguas residuales. 
En el Biofiltro experimental 1 se utilizó como empaque para el sistema de tratamiento; aserrín y viruta de madera, material muy empleado en sistemas de tratamiento por ser un recurso de fácil adquisición y bajo costo. Los resultados arrojaron que la eficiencia de este Biofiltro experimental fue de $53.53 \%$ en la remoción de contaminantes.

Las características físicas, químicas y biológicas del agua residual tratada en el Biofiltro experimental 1 (empaque de aserrín y viruta), de acuerdo con los informes de laboratorio, la mayoría de parámetros medidos alcanzaron valores fuera de la norma ambiental: $\mathrm{pH}=5.8$, Sólidos Suspendidos Totales $=370 \mathrm{mg} / \mathrm{l}$, Sólidos Totales $=3264 \mathrm{mg} / \mathrm{l}$, Colonias $=1 \times 10^{5} \mathrm{NMP} / 100$ $\mathrm{ml}, \mathrm{A} \& \mathrm{G}=0.3 \mathrm{mg} / \mathrm{l}, \mathrm{DQO}=3650 \mathrm{mg} / \mathrm{l}, \mathrm{DBO}=39 \mathrm{mg} / \mathrm{l}$, Hierro = $6.6 \mathrm{mg} / \mathrm{l}$, demostrando poca eficiencia de tratamiento de acuerdo con lo esperado en la investigación.

En el Biofiltro experimental 2 se utilizó como variante de empaque para el sistema de tratamiento; fibra de coco, que es remanente de la extracción de aceite y pulpa de coco que es un recurso natural renovable y orgánico. Los resultados arrojaron que la eficiencia de este Biofiltro experimental fue de $82.37 \%$ en la remoción de contaminantes.

Las características físicas, químicas y biológicas del agua residual tratada en el Biofiltro experimental 2 (empaque de fibra de coco), de acuerdo con los informes de laboratorio, los parámetros medidos alcanzaron valores dentro de la norma ambiental: $\mathrm{pH}=6.9$, Sólidos Suspendidos Totales $=40 \mathrm{mg} / \mathrm{l}$, Sólidos Totales $=603 \mathrm{mg} / \mathrm{l}$, Cloro $=0.1 \mathrm{mg} / \mathrm{l}$, Colonias $=1 \times 10^{5}$ $\mathrm{NMP} / 100 \mathrm{ml}, \mathrm{A} \& \mathrm{G}=0.3 \mathrm{mg} / \mathrm{l}, \mathrm{DQO}=174 \mathrm{mg} / \mathrm{l}, \mathrm{DBO}=23 \mathrm{mg} / \mathrm{l}$, Hierro = $0.19 \mathrm{mg} / \mathrm{l}$, demostrando alta eficiencia de tratamiento de acuerdo con lo esperado en la investigación.

Las lombrices seleccionadas en la investigación para la etapa de tratamiento del agua residual doméstica, se observó elevada tasa de mortalidad de lombrices adultas, 11 de 25 lombrices. Caso contrario sucedió con las lombrices jóvenes, 13 de 15 lombrices se adaptaron rápidamente a descomponer la materia orgánica presente en el agua residual.

\section{Bibliografía}

Arana, V. (2009). Guía para la toma de decisiones en la selección de sistemas de tratamiento de aguas residuales no convencionales. Lima, Perú: Avina.

Arango, J. (2003). Evaluación ambiental del Sistema Tohá en la remoción de Salmonella en aguas servidas domésticas. Universidad de Chile. Santiago de Chile, Chile: Biblioteca de la Universidad de Chile.

Carmona, C. (2010). Estudio del Comportamiento de una Mezcla de Aserrín y Grasa Láctea de Desecho. Universidad Austral de Chile. Valdivia, Chile: Biblioteca de la Universidad Austral de Chile. 
Castillo, F. (2005). Biotecnología Ambiental. Madrid, España: Tébar.

FAO. (2014). Agua residual. Recuperado el 10 de febrero de 2014, de sitio web de AQUASTAT: http://www.fao.org/nr/water/aquastat/data/glossary/search.html?lang=es\&_p=100\&submitBt $\mathrm{n}=-1$ \&keywords=Agua+residual\&subjectld=-1\&termld=-1\&submit=Buscar

Guzmán, M. (2004). Estudio de factibilidad de la aplicación del Sistema Tohá en la planta de tratamiento de aguas servidas de Valdivia. Universidad Austral de Chile. Valdivia, Chile: Biblioteca de la Universidad Austral de Chile.

Hernández, Y. (2005). Anteproyecto de construcción para aplicación de Lombricultura al tratamiento de planta Llau-Llao de Salmonera Invertec S.A. Universidad Austral de Chile. Valdivia, Chile: Biblioteca de la Universidad Austral de Chile.

Masters, G., \& Ela, W. (2008). Introducción a la ingeniería medioambiental. Madrid, España: Pearson.

Ministerio del Ambiente. (31 de marzo de 2003). Norma de calidad ambiental y descarga de efluentes: Recurso agua. Texto Unificado de Legislación Ambiental Secundaria del Ministerio del Ambiente. Quito, Ecuador.

Reckmann, O. (2011). Fibra de coco un sustrato con grandes ventajas. Redagrícola, 80.

Salazar, P. (2005). "Sistema Tohá; una alternativa ecológica para el tratamiento de aguas residuales en sectores rurales". Universidad Austral de Chile. Valdivia, Chile: Biblioteca de la Universidad Austral de Chile. 